\title{
GMR
}

\section{Using the attract method to identify core pathways in juvenile idiopathic arthritis}

\author{
J.S. Li ${ }^{1}$, X.L. Gao ${ }^{2}$, Y.R. Liu $^{3}$ and Y. Dong ${ }^{4}$ \\ ${ }^{1}$ Department of Rheumatology and Immunology, Binzhou People's Hospital, \\ Binzhou, Shandong Province, China \\ ${ }^{2}$ Department of Endocrinology, Binzhou People's Hospital, Binzhou, \\ Shandong Province, China \\ ${ }^{3}$ Department of Central Sterile Supply, Binzhou People's Hospital, \\ Binzhou, Shandong Province, China \\ ${ }^{4}$ Pathogenic Biology and Immunology Laboratory, Basic Medical College, \\ Mudanjiang Medical University, Mudanjiang, Heilongjiang Province, China \\ Corresponding author: Y. Dong \\ E-mail: dongyanmudanjiang@163.com
}

Genet. Mol. Res. 15 (3): gmr.15038331

Received December 22, 2015

Accepted April 8, 2016

Published August 12, 2016

DOI http://dx.doi.org/10.4238/gmr.15038331

Copyright (C) 2016 The Authors. This is an open-access article distributed under the terms of the Creative Commons Attribution ShareAlike (CC BY-SA) 4.0 License

\begin{abstract}
The aim of this study was to identify core pathways associated with juvenile idiopathic arthritis (JIA) using the attract method. Kyoto Encyclopedia of Genes and Genomes pathways were determined using the GSEA-ANOVA method, based on the gene expression data of JIA. Syn-expression groups within core attractor pathways were identified by hierarchical clustering. Correlated sets of genes exhibiting highly similar profiles to the syn-expression groups were identified and each correlated set was subjected to a gene ontology functional enrichment analysis to discover potentially shared biological themes. Based on a false-discovery rate $<0.05$, we identified 11 significant pathways were identified as potential attractors. Flag genes
\end{abstract}


or uninformative genes were removed and 5 discriminative pathways: the proteasome, ribosome, protein export, spliceosome, and Parkinson's disease pathways were identified. A final set of syn-expression groups with a consistent trend of relative expression of pathway-related genes was obtained; that is, the proteasome, ribosome, protein export, spliceosome, and Parkinson's disease pathways were composed of 2, $2,1,2$, and 3 clusters, respectively. Genes in each correlated set shared common roles, and changes at the pathway level were more likely to be real. In light of these, the attract method was able to on expand important context to find distinguishing expression patterns within pathways. This paper predicted that the functional themes involved in protein synthesis (such as proteasome, ribosome, spliceosome) were closely related to the progression of JIA, which might contribute to the detection of therapy target for JIA.

Key words: Juvenile idiopathic arthritis; Attract; Syn-expression group; Gene ontology

\section{INTRODUCTION}

Juvenile idiopathic arthritis (JIA), characterized by swelling or pain in the joints, or limitation in joint movement, is a non-infective, autoimmune inflammatory joint disorder affecting children aged $<16$ years for a duration of 6 weeks or more (Ravelli and Martini, 2007). The clinical heterogeneity of this disease is responsible for its varying courses and outcomes, such as self-limited arthritis with no long-term disability and destructive arthritis with severe disabilities (Lovell, 2006). Despite the recent advances in the treatment of JIA, its recurrence rate remains high, with $>40 \%$ of all patients carrying the disease over to adulthood (Bertilsson et al., 2013). Polyarticular JIA is a major subset of JIA involving a large number of joints, and with a tendency to worsen over time; therefore, this disease can lead to serious complications if left untreated (Davidson, 2000), specifically during early stages of the disease. However, lack of an effective and specific approach hinders the early diagnosis of polyarticular JIA. Therefore, a better understanding of the pathogenesis of JIA might help in the development of effective methods for early diagnosis and the identification of underlying therapeutic targets.

JIA is an autoimmune disorder whose pathogenesis is affected by various environmental and genetic factors. A previous study has indicated that interleukin-1 plays an important role in mediating the inflammatory cascade underlying systemic onset JIA (Pascual et al., 2005). Moreover, polymorphisms in the $U N C 13 D$ gene are believed to contribute to the development of systemic JIA (Hazen et al., 2008). In contrast, Tang et al. (2014) proved the lack of any correlation between polymorphisms in interferon regulatory factor-5 and JIA susceptibility, contradictory to the results seen in other autoimmune diseases. Genes are strictly regulated in cells to execute proper biological functions in response to the perturbations caused by phenotypic changes. Pathway analysis is currently the first-choice method to extract and explain the underlying biology of genes with decreased complexity and increased explanatory power.

Generally, significant genes exhibiting differential expression patterns between different conditions are identified using expression-based analytical methods, followed by a

Genetics and Molecular Research 15 (3): gmr.15038331 
post hoc application of knowledge (obtained from databases such as the Kyoto Encyclopedia of Genes and Genomes; KEGG) to identify potential functional interpretations (Kanehisa and Goto, 2000). Although this method can be used to annotate large datasets, several genes with patterns that are significantly correlated to the selected genes are not identified because of the non-inclusion of KEGG resources. Fortunately, attract, an approach described by Mar et al. (2011a), can expand on the context that is not considered in the traditional KEGG method. Moreover, attract has a larger impact on the pathway number, as well as the pathway relevance.

In this study, core pathways associated with the development and progression of JIA were identified using the attract method, by detecting coordinated genome-wide changes in the gene expression. This method can be summarized in the following four steps: identification of the core KEGG pathways with considerable differences in gene expression between JIA patients and normal subjects; screening of syn-expression groups within a core attractor pathway module; identification of gene sets that exhibit profiles that are highly similar to the syn-expression groups within a core attractor pathway module; and the isolation of potentially shared biological processes by functional enrichment analysis of each of the syn-expression groups. Here, we attempted to demonstrate the power of the attract method in extracting the genome-wide expression data of JIA, and in identifying the underlying mechanisms and therapeutic targets of JIA.

\section{MATERIAL AND METHODS}

\section{Microarray data}

In this study, genome-wide peripheral blood gene expression data in children with polyarticular JIA (Accession No.: E-GEOD-13849) was downloaded from the ArrayExpress database, based on the GPL570 platform of the [HG-U133_Plus_2] Affymetrix Human Genome U133 Plus 2.0 Array. The dataset E-GEOD-13849 was composed of samples obtained from 59 healthy children and 61 children with polyarticular JIA. The sample characteristics are presented in a previous study (Griffin et al., 2009). A total of 54,675 probes were obtained for subsequent analyses.

\section{Attractor analysis}

The attract method was employed to identify the core pathways in JIA (Mar, et al., 2011a). This method is essentially the inverse of traditional gene expression analysis approaches, and is comprised of four key steps: determining the core KEGG pathways showing significant differential expression between JIA and normal conditions; identifying different syn-expression groups within a core attractor pathway module; finding sets of genes that exhibit highly similar profiles to the syn-expression groups in an attractor pathway module; and functional enrichment analysis of each syn-expression group to discover potentially shared biological themes.

\section{KEGG pathway}

The core pathway modules were defined by KEGG and identified by GSEA-ANOVA,

Genetics and Molecular Research 15 (3): gmr.15038331 
which identified pathways showing the most differential expression changes between JIA and normal conditions. Briefly, 54,675 probes were subjected to the KEGG enrichment analysis, and were assigned to one or more KEGG pathways. Pathways with less than 5 probes were discarded and the pathway level was examined using GSEA-ANOVA, a gene set enrichment algorithm based on ANOVA.

In this approach, ANOVA model was fitted to each gene and the gene expression was modeled via a single factor representing the groups as different levels of this class. The $F$-statistics was computed for all genes using the ANOVA model. In this study, the $F$-statistic reflects the strength of association of the expression of a gene between JIA and normal conditions. That is, a large $F$-statistic was indicative of strong condition-specific changes, while small $F$-statistic exhibited minimal condition-specific expression changes. The informative genes were then identified based on the $F$-statistics.

Subsequently, a KEGG pathway enrichment analysis was performed using probes on the array. As a large $F$-statistic is indicative of strong condition-specific changes, a pathway comprising gene members with a large $F$-statistic presented accumulated condition-specific changes. This relationship was verified using a $t$-test, wherein a comparative analysis of $\log _{2}$-transformed $F$-statistics between the pathway distribution and global distribution was implemented. The resulting $\mathrm{P}$ values were adjusted using the Benjamini-Hochberg false discovery rate (FDR)-based method (Benjamini and Hochberg, 1995), and KEGG pathways with FDR $<0.05$ were classified as attractors.

\section{Syn-expression groups}

In this step, each remarkable and discriminative attractor pathway was decomposed into subsets of genes that shared similar expression patterns named "syn-expression groups", which was the name originally used by Niehrs and Pollet (1999). A syn-expression group is a cluster of functionally interacting genes whose expression is tightly coordinated, which could serve as a discriminating profile to summarize the differential expression across two conditions. Moreover, it might be a key determinant factor leading to disease development. Therefore, in this study, we identified the syn-expression groups by decomposing significant pathways into correlated subsets. Briefly, the LIMMA model was employed to remove genes exhibiting no significant changes in expression between the two conditions. The remaining genes were subjected to a hierarchical clustering model, based on the Pearson correlation coefficient distance measure, to decompose each significant pathway into correlated subsets or syn-expression groups. The optimal number of syn-expression groups was decided via an informativeness metric (Mar et al., 2011b). The average expression profiles of the synexpression groups were then analyzed.

\section{Correlated partners of syn-expression groups}

In this approach, the core attractor pathway modules and the syn-expression groups were deduced from information restricted to the KEGG sources. Consequently, these inferences were of high quality. However, this accuracy came at the expense of low coverage, as only a small proportion of the genome was ultimately described. Significantly, all genes with highly correlated patterns within the original data were extrapolated using the syn-expression

Genetics and Molecular Research 15 (3): gmr.15038331 
groups. Correlation coefficients between genes annotated to the syn-expression group and unannotated genes were calculated in each syn-expression group. In this study, a cutoff value of 0.85 was used to identify correlated partners of syn-expression groups on the chip that shared similar expression patterns to the syn-expression groups. Finally, correlated gene sets were constructed, which extended the analysis to the entire expression dataset by identifying genes highly correlated to the discriminating patterns (syn-expression groups).

\section{Functional enrichment analysis}

In this study, gene ontology (GO) functional analysis was implemented for each correlated partner to identify trends in common functions potentially shared by these genes. Significant terms were identified based on the following criteria: FDR $<0.05$ and counts $>10$.

\section{RESULTS}

\section{Identification of attractor pathway modules}

Based on the gene expression data obtained from healthy and polyarticular JIA samples, the core attractor pathways whose expression differed between the two groups were identified using the attract method. A total of 11 significantly enriched pathways with a threshold FDR value $<0.05$, believed to be attractors, were screened out, as depicted in Table 1 .

Table 1. Significant Kyoto Encyclopedia of Genes and Genomes (KEGG) pathways identified by attract that discriminate between two groups based on false discovery rates $(\mathrm{FDR})<0.05$.

\begin{tabular}{l|l|c|c}
\hline KEGG ID & KEGG term & FDR & Number of detected genes \\
\hline 3050 & Proteasome & $1.96 \mathrm{E}-07$ & 75 \\
\hline 4080 & Neuroactive ligand-receptor interaction & $1.24 \mathrm{E}-05$ & 508 \\
\hline 3010 & Ribosome & $4.43 \mathrm{E}-05$ & 141 \\
\hline 982 & Drug metabolism - cytochrome P450 & $1.14 \mathrm{E}-03$ & 119 \\
\hline 3060 & Protein export & $1.14 \mathrm{E}-03$ & 51 \\
\hline 3040 & Spliceosome & $5.47 \mathrm{E}-03$ & 287 \\
\hline 3018 & RNA degradation & $1.36 \mathrm{E}-02$ & 174 \\
\hline 5012 & Parkinson's disease & $1.85 \mathrm{E}-02$ & 228 \\
\hline 4740 & Olfactory transduction & $3.16 \mathrm{E}-02$ & 173 \\
\hline 190 & Oxidative phosphorylation & $3.68 \mathrm{E}-02$ & 232 \\
\hline 4020 & Calcium signaling pathway & $3.85 \mathrm{E}-02$ & 467 \\
\hline
\end{tabular}

\section{Identifying syn-expression groups}

Following the identification of candidate pathways, we categorized pathway-defined gene lists into highly correlated subgroups to highlight syn-expression groups reflecting gene sets responsible for the condition-specific differences. The informativeness metric was used to determine the optimal number of clusters. Removal of the flag genes or uninformative genes resulted in the isolation of 5 discriminative pathways (proteasome, ribosome, protein export, spliceosome, and Parkinson's disease). The syn-expression groups were isolated by decomposing each of these 5 significant, discriminative pathways into correlated subsets via hierarchical clustering. For example, the proteasome pathway was composed of 2 syn-

Genetics and Molecular Research 15 (3): gmr.15038331 
expression groups, which were composed of 11 and 6 correlated genes (Figure 1). Similarly, 2 syn-expression groups were identified for the ribosome pathway, composed of 24 and 8 correlated genes (Figure 2). The protein export pathway, however, was composed of only 1 synexpression group, comprising 17 correlated genes (Figure 3). We screened 2 syn-expression groups for the spliceosome pathway, comprising 11 and 47 correlated genes (Figure 4). Finally, we isolated 3 syn-expression groups from the Parkinson's disease pathways, comprising 6,10 , and 38 correlated subsets of genes (Figure 5). Accordingly, we found that the trend of the relative expression of pathway-related genes in these syn-expression groups was consistent. Therefore, we obtained unique syn-expression groups from each significant pathway, which reflected the pathway-specific expression patterns driven by a few genes.
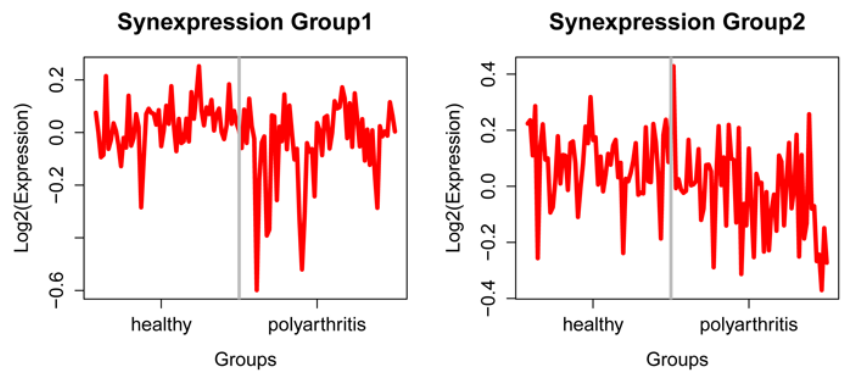

Figure 1. Average expression profiles of the syn-expression groups for the proteasome pathway. Sample categories are listed across the $\mathrm{X}$-axis and the $\log _{2}$ (expression) is presented across the Y-axis. Each inflection point represents the average gene expression in each sample within a group.
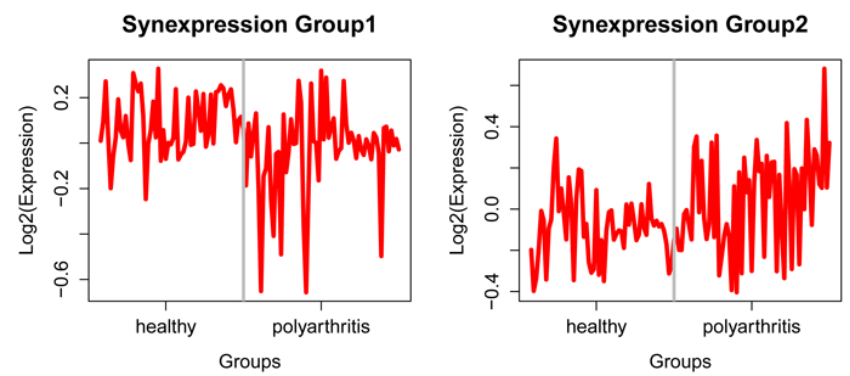

Figure 2. Average expression profiles of the syn-expression groups for the ribosome pathway.

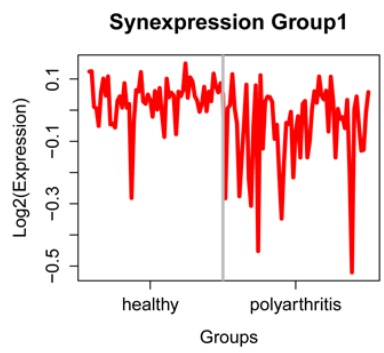

Figure 3. Average expression profiles of the syn-expression groups for the protein export pathway.

Genetics and Molecular Research 15 (3): gmr.15038331 

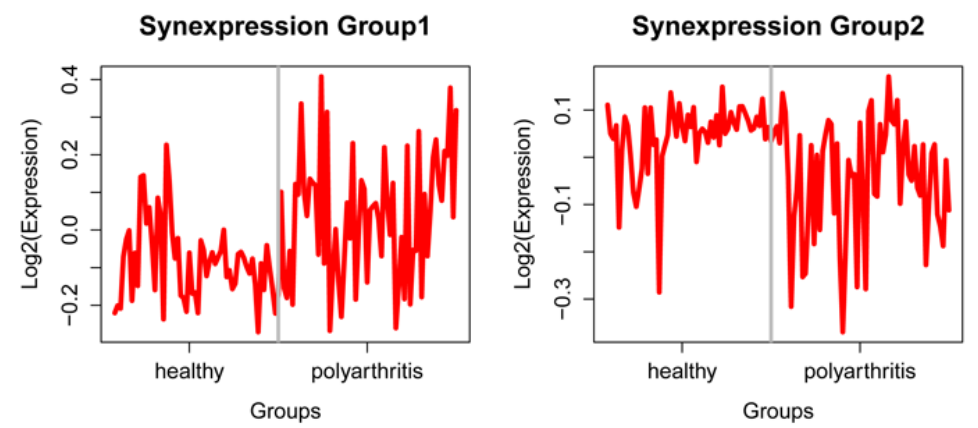

Figure 4. Average expression profiles of the syn-expression groups for the spliceosome pathway.
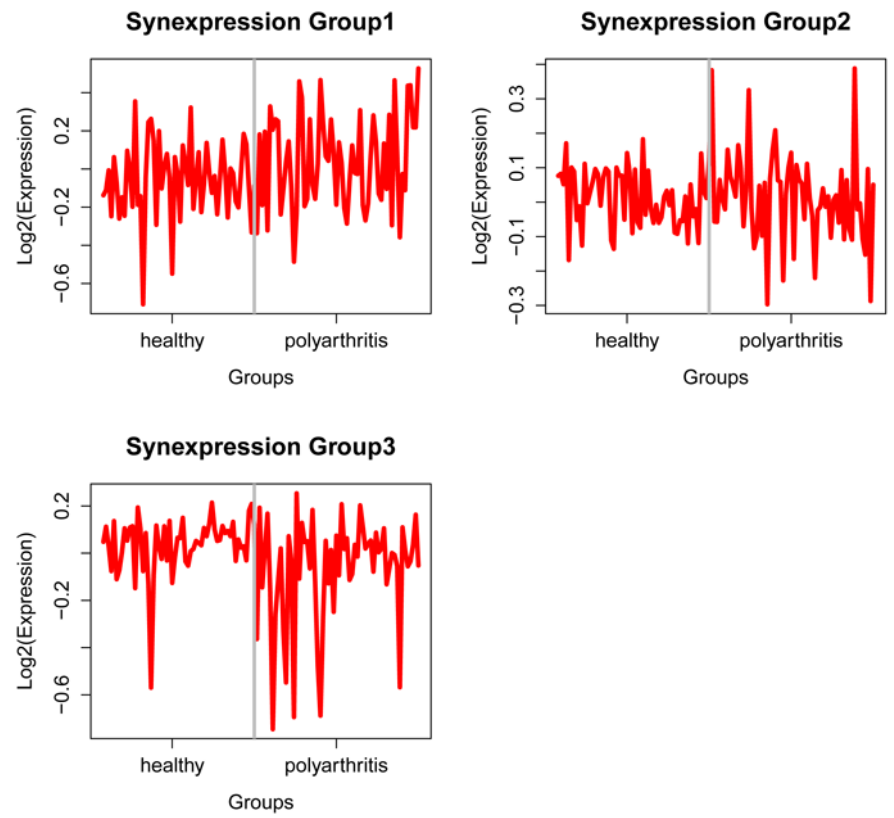

Figure 5. Average expression profiles of the syn-expression groups for pathways specific for Parkinson's disease.

\section{Identification of correlated partners of syn-expression groups}

In the initial GSEA steps of our study, genes with highly correlated patterns to the syn-expression groups were not included because of the lack of inclusion in KEGG resources. However, under a cutoff value (correlation coefficient) of 0.85, we identified 2, 2, 1, 2, and 3 clusters for the proteasome, ribosome, protein export, spliceosome, and Parkinson's disease pathways, respectively. Moreover, we discovered a total of 18, 72, 5, 51, and 161 unannotated genes for these pathways, respectively (see Figures $\mathbf{S 1}, \underline{\mathbf{S 2}}, \underline{\mathbf{S 3}}, \underline{\mathbf{S 4}}$, and $\underline{\mathbf{5}}$ ). Therefore, the attract method was able to expand important context, which was not seen in the traditional KEGG method. 


\section{GO functional enrichment analysis}

Significantly enriched GO terms with FDR $<0.05$ and counts $>10$ were identified for all pathways: 16 significant GO terms were identified in the proteasome pathway; genes were enriched in 22 significant functional terms in the ribosome pathway; and 20, 27, and 34 GO categories were identified for the protein export, spliceosome, and Parkinson's disease pathways, respectively. Of note, the two most significant GO terms of genes in correlated sets of the spliceosome pathway were catalytic step 2 spliceosome and spliceosomal complex. The genes in each correlated set shared common roles; accordingly, these genes belonged to the same GO term, and changes at the pathway level were more likely to be real.

\section{DISCUSSION}

In this study, the attract method was used to identify core pathways to explore the underlying molecular mechanism of JIA. Five core discriminative pathways (proteasome, ribosome, protein export, spliceosome, and Parkinson's disease pathways) were screened out, and the uniqueness of the syn-expression groups obtained from each core pathway, which reflected the pathway-specific expression patterns driven by a few genes, was confirmed. Accordingly, the genes in each syn-expression group belonged to the same GO term, which seemed to verify the authenticity of the pathway-level changes.

Expression-based analysis was developed to screen differentially expressed genes between experimental groups (Reiner et al., 2003). Subsequently, KEGG or meta-analysis is used to identify the underlying functions of the discovered genes. On the other hand, several genes might be ignored because of the lack of inclusion in the KEGG. Fortunately, attract, a modular process described by Mar et al. (2011a), has been shown to expand on important context that is not seen in traditional KEGG analyses. Moreover, attract has a larger impact on the pathway number, as well as the pathway relevance. Attract expands these deductions on these bases by identifying newly regulated coordinating genes that may be related to the mechanisms of disease. In agreement with this, expression-based analysis isolated only those correlated gene sets that exhibit profiles similar to the syn-expression groups; for example, $P P I B$, which is not a proteasome pathway-related gene, was not extracted. Therefore, we inferred that the attract method could help in the identification of crucial pathways and genes that could help uncover the disease mechanisms.

In this study, the proteasome pathway was identified as a core pathway of JIA using the attract method. Proteasomes, which form a major part of the ubiquitin-proteasome system (UPS), regulate several enzymatic activities that are responsible for controlling the gene expression and gene-environment interaction (Wang et al., 2007; Konstantinova et al., 2008). Specifically, insufficient proteasome function has also been reported to contribute to the pathophysiology of inflammatory disease (Zemeckienè et al., 2013). UPS (comprising ubiquitin and proteasome) has been shown to mediate the processing and degradation of a majority of regulatory proteins in eukaryotes. Moreover, UPS is the primary factor that activates the NF- $\mathrm{KB}$ signaling pathway (Hershko, 2005), which has been shown to play a major role in arthritis progression in animal models of inflammatory arthritis (Mor et al., 2005). Specifically, proteasome inhibitors are believed to inhibit NF- $\kappa B$ activity (Jana, 2008). However, proteasome inhibitors have been shown to induce osteoclast differentiation and

Genetics and Molecular Research 15 (3): gmr.15038331 
activate bone erosion, resulting in the development of chronic arthritis (Romas et al., 2000; Polzer et al., 2011). Accordingly, the proteasome pathway was theorized to play a core roles in JIA, probably by way of NF- $\mathrm{KB}$ signaling pathway regulation and bone destruction.

Attract also identified the spliceosome pathway as a significant pathway in JIA. Alternative splicing of genes is a fundamental and significant mechanism responsible for the regulation of gene function, and plays an important role in disease occurrence and development (Modrek et al., 2001). JIA is a well-known autoimmune and inflammatory joint disorder. Previous studies have reported a connection between the spliceosome-mediated autoimmune response and the development of rheumatoid arthritis (Hassfeld et al., 1995). In fact, Heinhuis et al. (2011) reported that splicing of IL-32 $\gamma$ into IL-32 $\beta$ exerts a protective role against inflammatory arthritis, by dampening the secretion of IL-32 $\gamma$, a potent cytokine responsible for enhanced inflammatory arthritis. Moreover, GO functional enrichment of genes in correlated sets, performed to verify the attract method, identified catalytic step 2 spliceosome and spliceosomal complex as the most significant GO terms. This strongly suggested that attract could be used to expand the significant context, and that the spliceosome pathway plays a major role in JIA by regulating the expression of inflammatory factors.

This study is subject to several drawbacks: the sample size was not large enough to affect the conclusions to a certain degree; the results obtained by bioinformatic analyses were not verified in vivo. However, despite these disadvantages, we believe that the attract method and the predicted core pathways (proteasome and spliceosome pathways) offer investigators with valuable resources to better understand the mechanisms of JIA, to detect biomarkers of novel underlying pathways, and identify drug targets for JIA therapy.

\section{Conflicts of interest}

The authors declare no conflicts of interest.

\section{ACKNOWLEDGMENTS}

Research not supported by grants from any specific public funding agencies.

\section{REFERENCES}

Benjamini Y and Hochberg Y (1995). Controlling the false discovery rate: a practical and powerful approach to multiple testing. J. R. Stat. Soc. B 57: 289-300.

Bertilsson L, Andersson-Gäre B, Fasth A, Petersson IF, et al. (2013). Disease course, outcome, and predictors of outcome in a population-based juvenile chronic arthritis cohort followed for 17 years. J. Rheumatol. 40: 715-724. http://dx.doi. org/10.3899/jrheum. 120602

Davidson J (2000). Juvenile idiopathic arthritis: a clinical overview. Eur. J. Radiol. 33: 128-134. http://dx.doi.org/10.1016/ $\underline{\mathrm{S} 0720-048 \mathrm{X}(99) 00161-8}$

Griffin TA, Barnes MG, Ilowite NT, Olson JC, et al. (2009). Gene expression signatures in polyarticular juvenile idiopathic arthritis demonstrate disease heterogeneity and offer a molecular classification of disease subsets. Arthritis Rheum. 60: 2113-2123. http://dx.doi.org/10.1002/art.24534

Hassfeld W, Steiner G, Studnicka-Benke A, Skriner K, et al. (1995). Autoimmune response to the spliceosome. An immunologic link between rheumatoid arthritis, mixed connective tissue disease, and systemic lupus erythematosus. Arthritis Rheum. 38: 777-785. http://dx.doi.org/10.1002/art.1780380610

Hazen MM, Woodward AL, Hofmann I, Degar BA, et al. (2008). Mutations of the hemophagocytic lymphohistiocytosisassociated gene UNC13D in a patient with systemic juvenile idiopathic arthritis. Arthritis Rheum. 58: 567-570. http://

Genetics and Molecular Research 15 (3): gmr.15038331 
dx.doi.org/10.1002/art.23199

Heinhuis B, Koenders MI, van de Loo FA, Netea MG, et al. (2011). Inflammation-dependent secretion and splicing of IL$32 \gamma$ in rheumatoid arthritis. Proc. Natl. Acad. Sci. USA 108: 4962-4967. http://dx.doi.org/10.1073/pnas.1016005108

Hershko A (2005). The ubiquitin system for protein degradation and some of its roles in the control of the cell division cycle. Cell Death Differ. 12: 1191-1197. http://dx.doi.org/10.1038/sj.cdd.4401702

Jana NR (2008). NSAIDs and apoptosis. Cell. Mol. Life Sci. 65: 1295-1301. http://dx.doi.org/10.1007/s00018-008-7511-x

Kanehisa M and Goto S (2000). KEGG: kyoto encyclopedia of genes and genomes. Nucleic Acids Res. 28: 27-30. http:// dx.doi.org/10.1093/nar/28.1.27

Konstantinova IM, Tsimokha AS and Mittenberg AG (2008). Role of proteasomes in cellular regulation. Int. Rev. Cell Mol. Biol. 267: 59-124. http://dx.doi.org/10.1016/S1937-6448(08)00602-3

Lovell DJ (2006). Update on treatment of arthritis in children: new treatments, new goals. Bull. NYU Hosp. Jt. Dis. 64: $72-76$.

Mar JC, Matigian NA, Quackenbush J and Wells CA (2011a). attract: A method for identifying core pathways that define cellular phenotypes. PLoS One 6: e25445. http://dx.doi.org/10.1371/journal.pone.0025445

Mar JC, Wells CA and Quackenbush J (2011b). Defining an informativeness metric for clustering gene expression data. Bioinformatics 27: 1094-1100. http://dx.doi.org/10.1093/bioinformatics/btr074

Modrek B, Resch A, Grasso C and Lee C (2001). Genome-wide detection of alternative splicing in expressed sequences of human genes. Nucleic Acids Res. 29: 2850-2859. http://dx.doi.org/10.1093/nar/29.13.2850

Mor A, Abramson SB and Pillinger MH (2005). The fibroblast-like synovial cell in rheumatoid arthritis: a key player in inflammation and joint destruction. Clin. Immunol. 115: 118-128. http://dx.doi.org/10.1016/j.clim.2004.12.009

Niehrs C and Pollet N (1999). Synexpression groups in eukaryotes. Nature 402: 483-487. http://dx.doi.org/10.1038/990025

Pascual V, Allantaz F, Arce E, Punaro M, et al. (2005). Role of interleukin-1 (IL-1) in the pathogenesis of systemic onset juvenile idiopathic arthritis and clinical response to IL-1 blockade. J. Exp. Med. 201: 1479-1486. http://dx.doi. org/10.1084/jem.20050473

Polzer K, Neubert K, Meister S, Frey B, et al. (2011). Proteasome inhibition aggravates tumor necrosis factor-mediated bone resorption in a mouse model of inflammatory arthritis. Arthritis Rheum. 63: 670-680. http://dx.doi.org/10.1002/ art.30177

Ravelli A and Martini A (2007). Juvenile idiopathic arthritis. Lancet 369: 767-778. http://dx.doi.org/10.1016/S01406736(07)60363-8

Reiner A, Yekutieli D and Benjamini Y (2003). Identifying differentially expressed genes using false discovery rate controlling procedures. Bioinformatics 19: 368-375. http://dx.doi.org/10.1093/bioinformatics/btf877

Romas E, Bakharevski O, Hards DK, Kartsogiannis V, et al. (2000). Expression of osteoclast differentiation factor at sites of bone erosion in collagen-induced arthritis. Arthritis Rheum. 43: 821-826. http://dx.doi.org/10.1002/15290131(200004)43:4<821::AID-ANR12>3.0.CO;2-T

Tang L, Chen B, Ma B and Nie S (2014). Association between IRF5 polymorphisms and autoimmune diseases: a metaanalysis. Genet. Mol. Res. 13: 4473-4485. http://dx.doi.org/10.4238/2014.June.16.6

Wang X, Guerrero C, Kaiser P and Huang L (2007). Proteomics of proteasome complexes and ubiquitinated proteins. Expert Rev. Proteomics 4: 649-665. http://dx.doi.org/10.1586/14789450.4.5.649

Zemeckienė Z, Vitkauskienė A, Sjakste T, Sitkauskienė B, et al. (2013). Proteasomes and proteasomal gene polymorphism in association with inflammation and various diseases. Medicina (Kaunas) 49: 207-213.

\section{Supplementary material}

Figure S1. Average expression profiles of the correlated set of genes in the proteasome pathway. Gene clusters 1 and 2 were composed of 18 and 0 correlated genes, respectively. The X-and Y-axes listed the sample categories and $\log _{2}$ (expression), respectively. Each inflection point reflects the average gene expression of each sample within a group.

Figure S2. Average expression profiles of the correlated set of genes in the ribosome pathway. Gene clusters 1 and 2 were composed of 72 and 0 correlated genes, respectively.

Figure S3. Average expression profiles of the correlated set of genes in the protein export pathway. The cluster was composed of 5 correlated genes.

Genetics and Molecular Research 15 (3): gmr.15038331 
Figure S4. Average expression profiles of the correlated sets of genes in the splicesome pathway. The clusters 1 and 2 were composed of 0 and 51 correlated genes, respectively.

Figure S5. Average expression profiles of the correlated sets of genes in the Parkinson's disease pathways. Gene clusters 1,2 , and 3 were composed of 62,0 , and 99 correlated genes, respectively. 